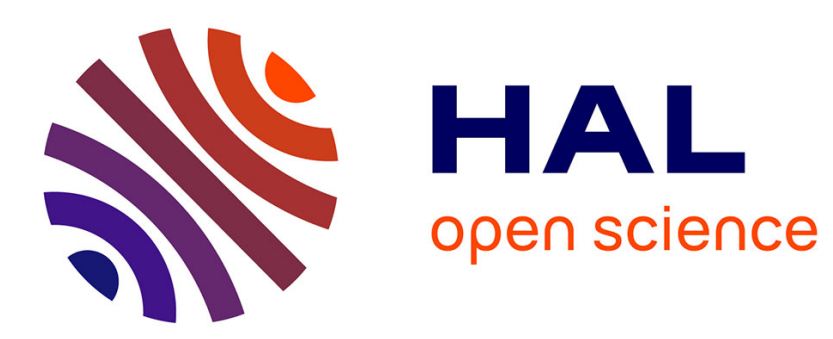

\title{
Closed-loop Control of a Novel 2-DOF MEMS Nanopositioner with Electrothermal Actuation.
}

\author{
Anthony Fowler, Micky Rakotondrabe, S.O. Reza Moheimani
}

\section{To cite this version:}

Anthony Fowler, Micky Rakotondrabe, S.O. Reza Moheimani. Closed-loop Control of a Novel 2-DOF MEMS Nanopositioner with Electrothermal Actuation.. The 6th IFAC Symposium on Mechatronic Systems (Mechatronics'2013), Jan 2013, China. pp.391-398. hal-00841804

\section{HAL Id: hal-00841804 https://hal.science/hal-00841804}

Submitted on 5 Jul 2013

HAL is a multi-disciplinary open access archive for the deposit and dissemination of scientific research documents, whether they are published or not. The documents may come from teaching and research institutions in France or abroad, or from public or private research centers.
L'archive ouverte pluridisciplinaire HAL, est destinée au dépôt et à la diffusion de documents scientifiques de niveau recherche, publiés ou non, émanant des établissements d'enseignement et de recherche français ou étrangers, des laboratoires publics ou privés. 


\title{
Closed-loop Control of a Novel 2-DOF MEMS Nanopositioner with Electrothermal Actuation *
}

\author{
Anthony G. Fowler* Micky Rakotondrabe** \\ S. O. Reza Moheimani* \\ * School of Electrical Engineering and Computer Science, \\ University of Newcastle, Callaghan, NSW 2308, Australia \\ (e-mail: Anthony.Fowler@uon.edu.au, \\ Reza.Moheimani@newcastle.edu.au). \\ ** FEMTO-ST Institute, UMR CNRS 6174 - UFC / ENSMM / \\ UTBM, Automatic Control and Micro-Mechatronic Systems depart., \\ AS2M, 25000 Besançon, France (e-mail: mrakoton@femto-st.fr)
}

\begin{abstract}
The design, characterization and control of a novel 2-DoF MEMS nanopositioner is presented, with Z-shaped electrothermal actuators being used to position the device's central stage. Whereas the more commonly-used V-shaped electrothermal actuator only allows displacements in one direction, the design of the Z-shaped beams used in the presented device allows two actuators to be coupled back-to-back to achieve bidirectional motion along each of the two axes. Testing of the device shows that stage displacements in excess of $\pm 5 \mu \mathrm{m}$ are achievable for both the $x$ and $y$ axes. The device features integrated displacement sensors based on polysilicon electrothermal heaters, which are supplied with an electrical bias voltage that results in Joule heating. The resistance of each heater varies depending on the position of the central stage, with two heaters being used per axis in a differential configuration. The displacement measurements are utilized as part of an implemented closed-loop control scheme that uses both feedforward and feedback mechanisms based on the principle of internal model control. Experimental testing shows that the use of the controller enhances the static and dynamic performance of the system, with particular improvements being seen in the device's reference tracking, response time and cross-coupling rejection.
\end{abstract}

Keywords: MEMS, nanopositioning, electrothermal actuator, electrothermal sensor, feedforward, feedback

\section{INTRODUCTION}

Nanopositioning systems have proven to be highly invaluable in many fields of micro and nanotechnology due to their ability to provide high-precision motion with nanometer resolution. A particularly important application is the atomic force microscope (AFM) (Binnig and Rohrer (1986)), which uses a nanopositioner to position a sample while measurement's of the sample's topography are made using a microcantilever with a sharp probe. The need for precise mechanical positioning has also seen nanopositioning systems used in novel probe-based data storage systems (Pantazi et al. (2007); Lantz et al. (2007)).

There is a growing interest in the use of microelectromechanical systems (MEMS) fabrication processes to create miniaturized nanopositioners. These devices provide a number of potential advantages over comparable macroscale versions including having a smaller footprint, increased bandwidth, lower bulk fabrication costs and easier mass fabrication (Bergna et al. (2005); Zhu et al. (2011b)). A MEMS nanopositioner has recently been demonstrated

\footnotetext{
^ This work was supported by the Australian Research Council.
}

in a AFM application (Fowler et al. (2012)), where the device was used to replace the existing scanning stage of a commercial AFM. The nanopositioner's stage was fabricated with a series of integrated $3 \mu \mathrm{m}$ diameter gold features which were used to represent a scan sample, with the AFM being successfully used to perform an open-loop scan of the features in tapping mode.

This paper presents a novel 2-degree of freedom (DoF) MEMS nanopositioner that uses electrothermal actuation to position a central stage. Electrothermal actuators are a commonly-used mechanism in MEMS devices, maintaining a small footprint while providing high actuation forces and being relatively straightforward to fabricate (Guan and Zhu (2010); Chen and Culpepper (2012)). One of the most common types of electrothermal actuator is the $\mathrm{V}$ shaped, or "chevron" type actuator, which has been used in a range of MEMS applications (Chow and Lai (2009); Waterfall et al. (2008)). This actuator utilizes the thermal expansion of a series of bent beams to create a mechanical displacement, with the motion taking place in the direction towards which the beams are angled. The nanopositioner presented in this paper uses a relatively new type of electrothermal actuator that features Z-shaped beams, as 

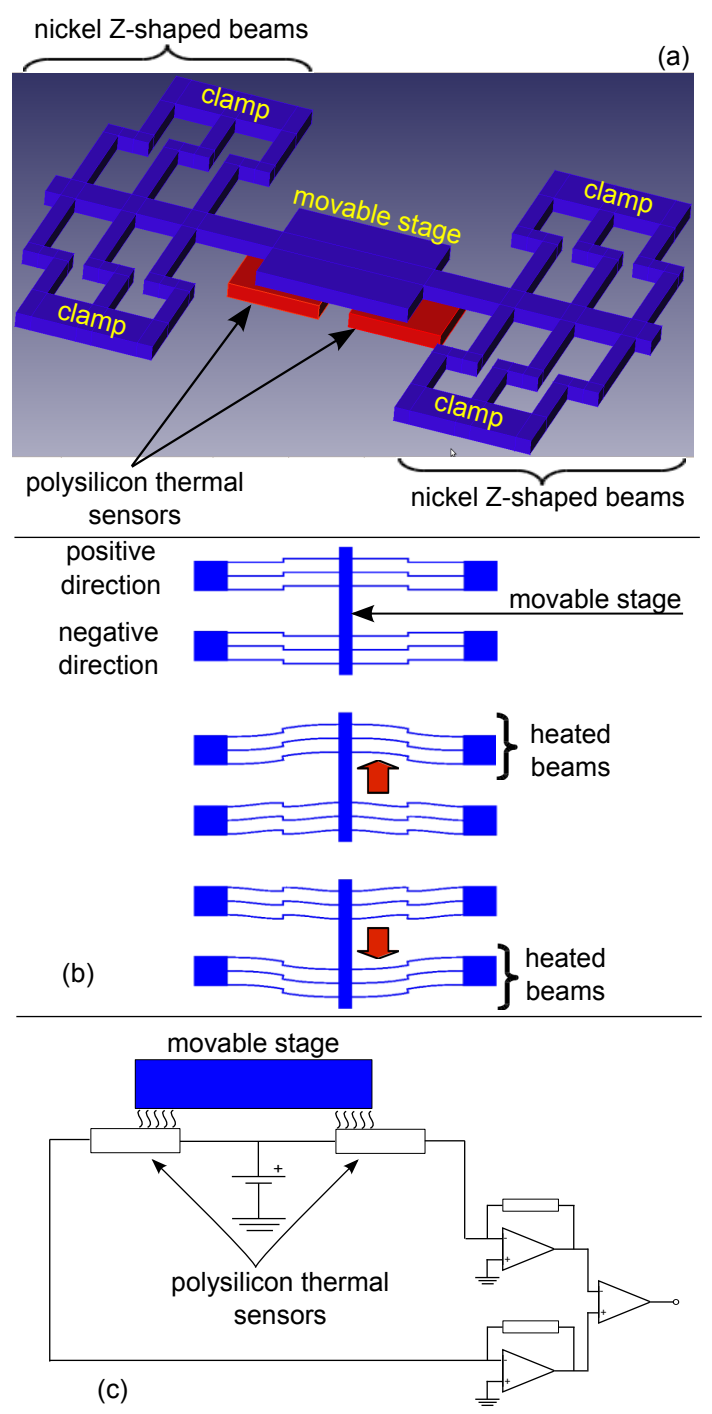

Fig. 1. Simplified schematic diagram of one axis of the nanopositioner.

demonstrated in Ouyang and Zhu (2012); Zhu et al. (2012). While still using the principle of thermal expansion, a significant advantage of this type of actuator is that bidirectional motion is made possible by coupling two actuators in a back-to-back configuration. This is not possible to achieve with the $\mathrm{V}$-shaped beam actuator due to its mechanical stiffness in the reverse direction.

The nanopositioner also features integrated electrothermal displacement sensors that are used to measure the displacement of the stage along each of the axes of the device. The principle of electrothermal sensing has been demonstrated in a range of MEMS devices (Pantazi et al. (2004); Zhu et al. (2011a)), and is a compact solution that allows for position measurements with sub-nanometer resolution (Lantz et al. (2005)) These displacement measurements are used to enable closed-loop control of the system, reducing cross-coupling and improving the static and dynamic performance of the device.

\section{NANOPOSITIONER DESIGN}

A simplified schematic diagram of the nanopositioner is shown in Fig. 1a. Each axis uses two Z-shaped electrother-

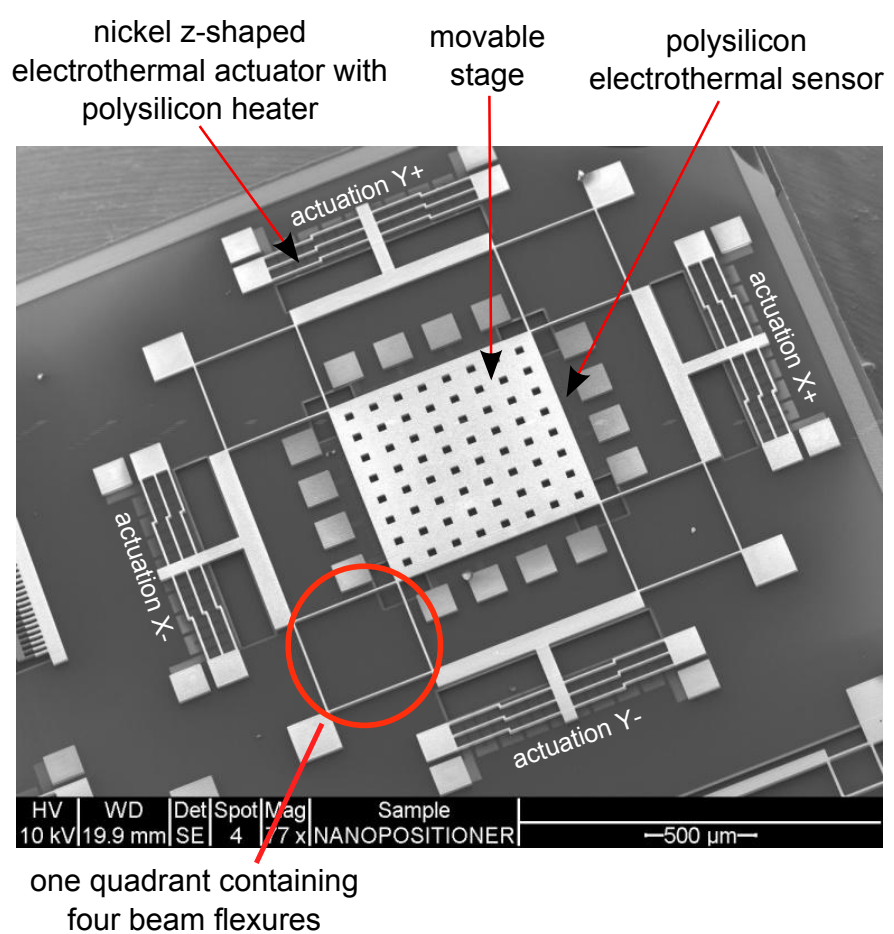

Fig. 2. SEM image of the fabricated nanopositioner.

mal actuators to provide bidirectional mechanical displacement of the central stage, and features an electrothermal sensor underneath the stage to act as an integrated displacement sensing mechanism.

\subsection{Actuation Principle}

Each of the two electrothermal actuators consists of a series of Z-shaped nickel beams and a polysilicon heater located underneath the beams. When an electrical current is passed through the heater, thermal energy is transferred via a small air gap to the Z-shaped beams. The resulting increase in the temperature of the beams results in thermal expansion, which creates a mechanical displacement that moves the central stage. A single actuator is used to provide displacement in a single direction (i.e. a positive or negative displacement), with the two actuators in each axis being mechanically connected back-to-back to provide the ability to achieve bidirectional displacements (as shown in Fig. 1b).

\subsection{Sensing Principle}

The integrated displacement sensor for each axis consists of a pair of polysilicon heaters positioned underneath the edge of the central stage. A constant bias voltage is applied to the heaters, with Joule heating causing the heaters to reach a temperature of several hundred degrees Celsius. This difference in temperature between the heaters and the stage results in the conduction of heat towards the stage via a small air gap (as shown in Fig. 1c). Changes in the position of the stage vary the overlapping area between the stage and the heaters, which affects the rate of heat conduction between the two bodies. This creates small fluctuations in the temperature of each heater, and due to the temperature-dependent resistivity of silicon, this produces measurable changes in the resistance of each 

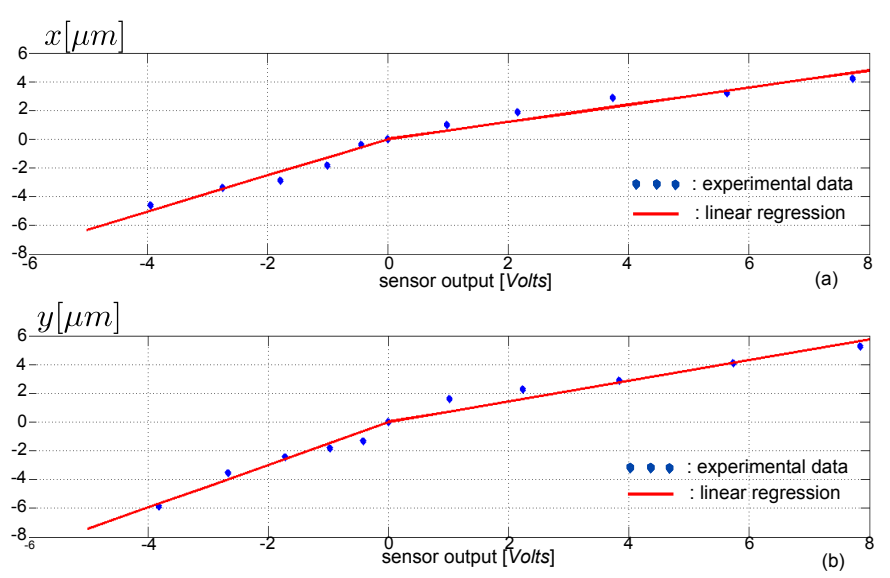

Fig. 3. Stage displacement versus sensor output for $x$ and $y$ axes.

heater. These resistance variations are converted into a detectable voltage which is amplified to produce a signal that is representative of the displacement of the stage. The two heaters per axis are employed in a differential configuration, which improves factors such as the linearity of the sensor output, and helps to reduce the influence of undesirable factors such as ambient temperature and sensor aging (Zhu et al. (2011b); Sebastian (2011)).

\subsection{2-DoF Layout and Fabrication}

The nanopositioner has two mechanical degrees of freedom, with both the $x$ and $y$ axes being designed to be mechanically identical. The device's central stage is approximately $1 \mathrm{~mm} \times 1 \mathrm{~mm}$ in size, with four electrothermal actuators being used to create displacements along both directions of each in-plane axis. Beam flexures located at the corners of the stage are designed to guide the stage's mechanical motion and to minimize the cross-coupling between the $x$ and $y$ axes during the operation of the device. In order to ensure that the transfer of heat from one actuator to another or to the stage is as small as possible, the flexures were designed with small cross-section dimensions of $8 \mu \mathrm{m} \times 20 \mu \mathrm{m}$ (width $\times$ height).

The 2-DoF nanopositioner was fabricated using the commercial MetalMUMPs process provided by MEMSCAP. The main structures of the device including the stage, actuator beams and flexures are made from $20 \mu \mathrm{m}$-thick electroplated nickel, while the electrothermal heaters for the actuators and sensors are made from $0.7 \mu \mathrm{m}$-thick polysilicon (Cowen et al. (2009)). The entire device occupies a die area of approximately $3 \mathrm{~mm} \times 3 \mathrm{~mm}$. A scanning electron microscope (SEM) image of the fabricated nanopositioner is shown in Fig. 2.

\section{EXPERIMENTAL TESTING AND CHARACTERIZATION}

\subsection{Electrothermal Displacement Sensor Characterization}

The relationship between the output of the nanopositioner's displacement sensors and the stage displacement was first characterized. A series of voltage steps of varying amplitudes were applied to one of the device's actuators at a time, while the in-plane displacement of the stage
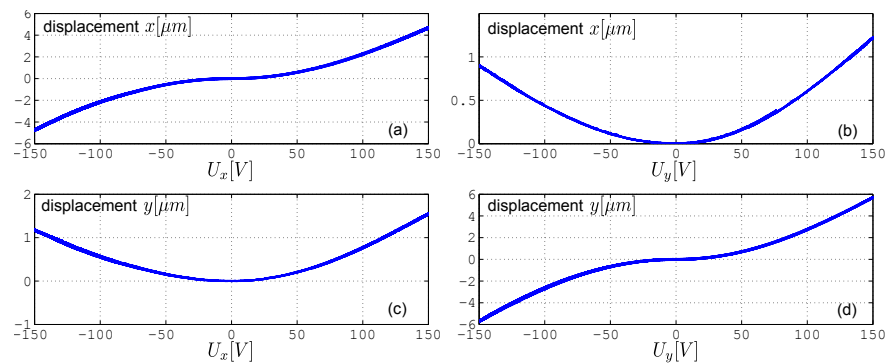

Fig. 4. Stage displacement versus actuation voltage for $x$ and $y$ axes.

was measured using a Polytec MSA-400 Micro System Analyzer (MSA). The voltage output of the sensing circuit for each axis was simultaneously recorded.

The resulting relationship between stage displacement and sensor output is shown in Fig. 3. As depicted in the figure, linear functions can be fitted to the experimental data to approximate the behavior of the sensors. It is evident however that the gradients of the fitted linear functions differ between the negative and positive displacements for each axis. This is possibly due to non-ideal placement of the two electrothermal heaters underneath the stage in the fabricated device, leading to asymmetrical operation of the sensors with respect to stage displacements in positive or negative directions. This asymmetry can be compensated for through the use of appropriate inverse gains that enable the displacement of the stage to be determined from measurements of the sensor outputs.

\subsection{Electrothermal Actuator Characterization}

Following the sensor calibration process described above, the relationship between the stage displacement and the actuation voltages applied to the nanopositioner's electrothermal actuators was obtained. A triangle wave $U_{x}$ with a frequency of $0.05 \mathrm{~Hz}$ was first applied to the nanopositioner's $x$ axis actuators, while the $y$ axis was supplied with $U_{y}=0 \mathrm{~V}$. Measurements of the stage displacement were obtained via the integrated electrothermal displacement sensors, providing both the input-output relationship for the $x$ axis as well as the mechanical cross-coupling between the axes. The actuating signals were then swapped to obtain the corresponding data for the $y$ axis.

The experimental results are displayed in Fig. 4. As shown, the device is able to produce stage displacements in excess of $\pm 5 \mu \mathrm{m}$ for both the $x$ and $y$ axes at the maximum applied actuation voltage of $150 \mathrm{~V}$. It is evident that there is a nonlinear relationship between the actuation voltage and the stage displacement, which is largely due to the quadratic relationship between the power delivered to the electrothermal actuators and the input voltage. Another possible factor is related to nonlinear phenomena involving the resistivity and thermal coefficients of the polysilicon comprising the device's actuators and sensors (Bazaei et al. (2012)). There is also some cross-coupling present between the $x$ and $y$ axes, which is most likely due to thermal leakage from an active actuator to the beams of the actuators in the other axes. As shown later in this paper, feedback control can be used to compensate for these nonlinearities and cross-coupling. 

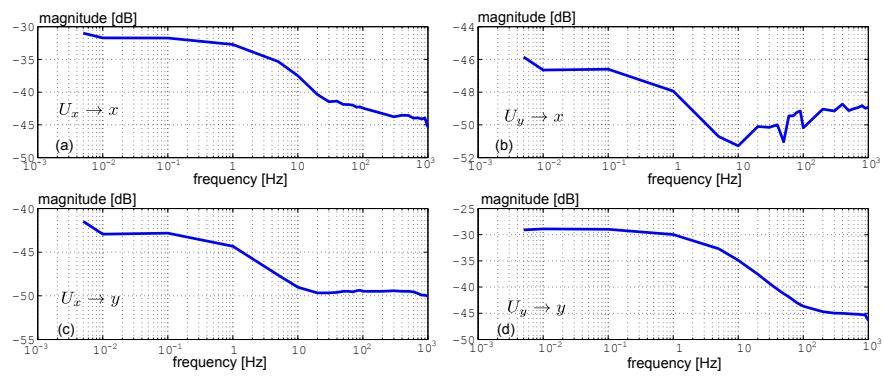

Fig. 5. Magnitude plots for experimental frequency response analysis.

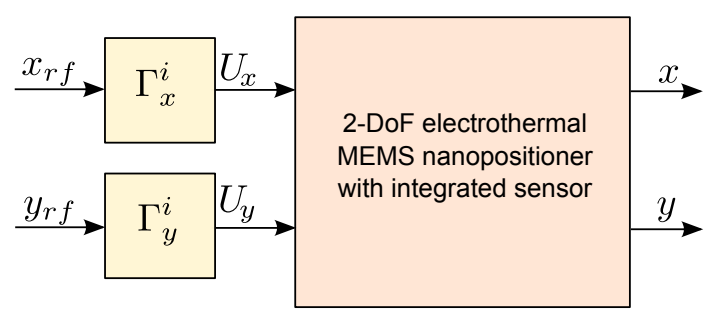

Fig. 6. Block diagram of system and feedforward controllers.
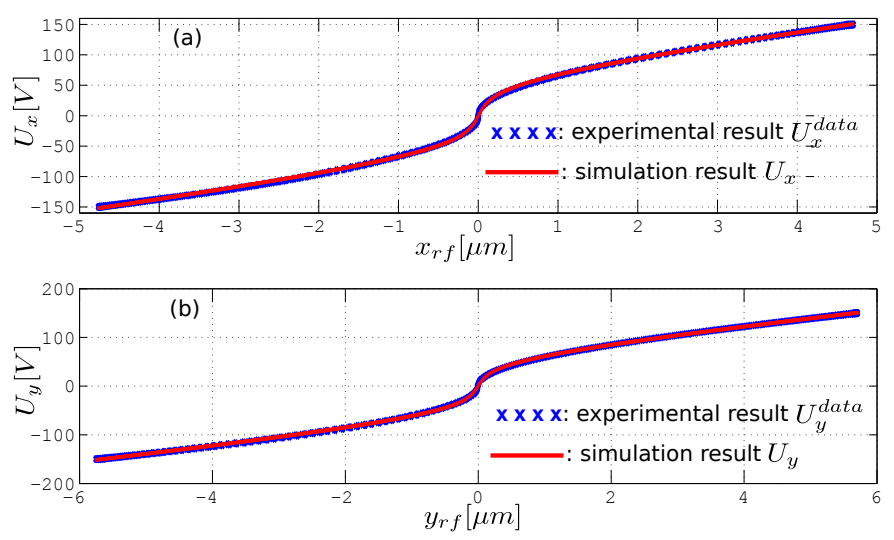

Fig. 7. Feedforward compensators $U_{x}(k)=\Gamma_{x}^{i}\left(x_{r f}(k)\right)$ and $U_{y}(k)=\Gamma_{y}^{i}\left(y_{r f}(k)\right)$. A comparison of experimental result and the simulated result is shown.

\subsection{Frequency Response}

The dynamic characteristics of the fabricated nanopositioner were evaluated by performing a frequency response analysis, with the results being shown in Fig. 5. These results indicate that the bandwidth of both the $x$ and $y$ axes is approximately $3 \mathrm{~Hz}$.

\section{FEEDFORWARD CONTROL AND MODELING}

\subsection{Feedforward Controller Design and System Linearization}

Feedforward controllers were designed in order to create a linear system by compensating for the static nonlinearities of the nanopositioner's electrothermal actuators. The block diagram in Fig. 6 shows the feedforward controllers for the $x$ and $y$ axes denoted by $\Gamma_{x}^{i}$ and $\Gamma_{y}^{i}$, with $x_{r f}$ and $y_{r f}$ representing the inputs to the system.

The feedforward compensators were calculated by constructing the maps $\left(x, U_{x}\right)$ and $\left(y, U_{y}\right)$ from the experimental data and deriving the feedforward controllers by letting $x_{r f}=x$ and $y_{r f}=y$. The resulting maps $\left(x_{r f}, U_{x}\right)$ and $\left(y_{r f}, U_{y}\right)$ are shown in Fig. 7. In order to approximate this mapping, a controller structure based on a hyperbolic arcsine function is proposed. This is given by the following generalized structure:

$$
\begin{gathered}
\left\{\begin{array}{l}
U_{x}(k)=\Gamma_{x}^{i}\left(x_{r f}(k)\right) \\
U_{y}(k)=\Gamma_{y}^{i}\left(y_{r f}(k)\right)
\end{array}\right. \\
\left\{\begin{array}{l}
U_{x}(k)=c_{1 x} x_{r f}(k)+c_{2 x} \sinh ^{-1}\left(c_{3 x} x_{r f}(k)+c_{4 x}\right) \\
U_{y}(k)=c_{1 y} y_{r f}(k)+c_{2 y} \sinh ^{-1}\left(c_{3 y} y_{r f}(k)+c_{4 y}\right)
\end{array}\right.
\end{gathered}
$$

where $c_{1 j}(j=x, y)$ is a coefficient for the affine term of the structure and $c_{2 j}, c_{3 j}$ and $c_{4 j}$ are the coefficients for the hyperbolic arcsine term. The affine term is introduced in order to improve the accuracy of the compensating controllers at the upper range of the inputs $x_{r f}$ and $y_{r f}$. The hyperbolic arcsine model alone is very accurate for low range inputs (within $\pm 1 \mu \mathrm{m}$ ), with reduced accuracy outside this range being compensated for by the addition of the affine term.

The identification of the coefficients of the controllers was carried out with the following least-square based optimization problem:

$$
\left\{\begin{array}{l}
\min _{\left(c_{1 x}, c_{2 x}, c_{3 x}, c_{4 x}\right) \in \mathbb{R}^{4}} \sum_{k=1}^{N_{\text {exp }}}\left(U_{x}^{\text {data }}(k)-U_{x}(k)\right)^{2} \\
\min _{\left(c_{1 y}, c_{2 y}, c_{3 y}, c_{4 y}\right) \in \mathbb{R}^{4}} \sum_{k=1}^{N_{\text {exp }}}\left(U_{y}^{\text {data }}(k)-U_{y}(k)\right)^{2}
\end{array}\right.
$$

where $U_{x}(k)$ and $U_{y}(k)$ are the outputs of the compensators as defined in (1). These simulated compensator models use the experimental displacement $x_{r f}(k)=x(k)$ and $y_{r f}(k)=y(k)$ as inputs. $U_{x}^{\text {data }}$ and $U_{y}^{\text {data }}$ are the experimentally-measured actuator voltages and $N_{\exp }$ is the length of the experimental data. Following identification, the coefficients are determined as follows:

$$
\left(\begin{array}{l}
c_{1 x} \\
c_{2 x} \\
c_{3 x} \\
c_{4 x}
\end{array}\right)=\left(\begin{array}{c}
16.4 \\
14.65 \\
16.17 \\
0.029
\end{array}\right) ; \quad\left(\begin{array}{l}
c_{1 y} \\
c_{2 y} \\
c_{3 y} \\
c_{4 y}
\end{array}\right)=\left(\begin{array}{l}
13.77 \\
14.19 \\
14.14 \\
0.057
\end{array}\right)
$$

A comparison of the compensators' simulated output and the experimental data is shown in Fig. 7, with a close match between the two being evident.

\subsection{Experimental Linearization Results}

The feedforward compensators were implemented according to the block diagram in Fig. 6 , with the linearity of the compensated system then being experimentally tested. A series of reference inputs $x_{r f}$ and $y_{r f}$ were applied to the electrothermal actuators of the $x$ and $y$ axis respectively, with the displacement of the stage being measured using the MSA. The resulting experimental data is shown in Fig. 8. As shown, the feedforward controllers are effective in compensating for the actuators' static nonlinearity, with the plotted displacement relationships possessing slopes close to $\frac{1 \mu \mathrm{m}}{1 \mu \mathrm{m}}$. It is noted that there is still some crosscoupling present between axes, however a proposed feedback controller will later be used to reduce this effect. 

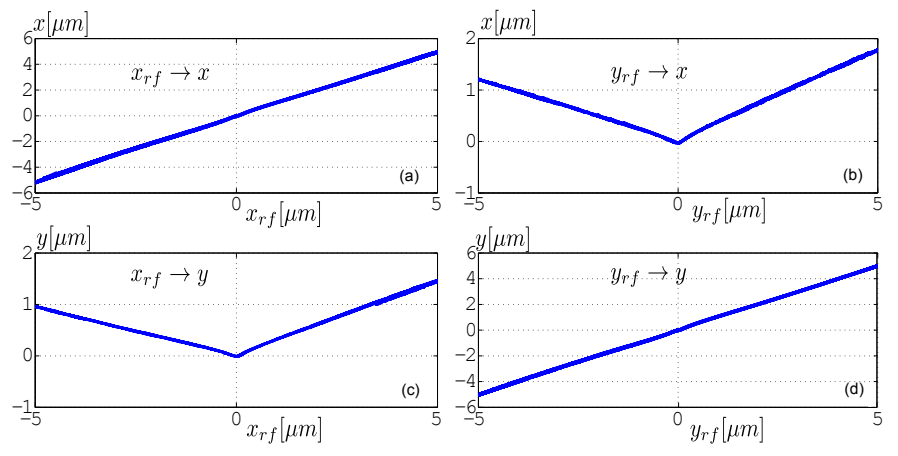

Fig. 8. Stage displacement versus reference displacement using feedforward compensators.
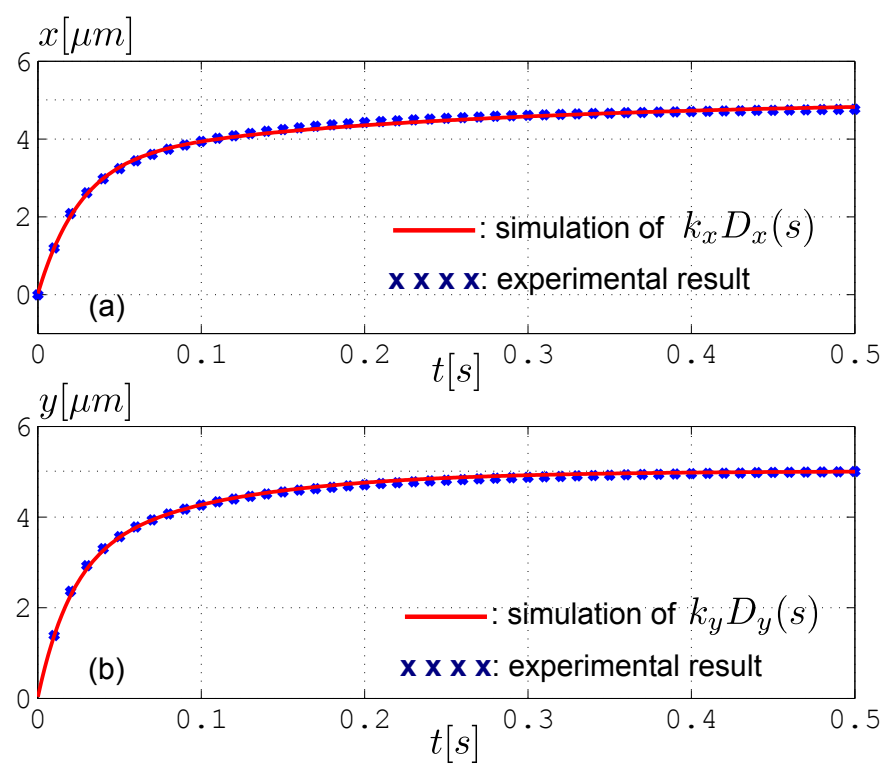

Fig. 9. Step responses for the compensated system. (a) $x_{r f}=5 \mu \mathrm{m}$. (b) $y_{r f}=5 \mu \mathrm{m}$.

\subsection{Modeling the Linearized System}

The compensated system, consisting of the fabricated 2DoF nanopositioner cascaded with the feedforward controllers, was modeled to facilitate the implementation of a closed-loop control system. The static characteristics of the system are given by the data presented in Fig. 8, while its dynamic characteristics can be identified from an experimental step response or frequency response. It is proposed that the mechanical cross-coupling between axes are treated as disturbances. The following model is used to represent the system:

$$
\left\{\begin{array}{l}
x=k_{x} D_{x}(s) x_{r f}+d_{x} \\
y=k_{y} D_{y}(s) y_{r f}+d_{y}
\end{array}\right.
$$

where $k_{x}$ and $k_{y}$ are the static gains of the system as identified from Figs. 8a and 8d respectively, while $d_{x}$ and $d_{y}$ are the cross-couplings $y_{r f} \rightarrow x$ and $x_{r f} \rightarrow y$ respectively. Note that these cross-couplings include both a static component identifiable from Figs. 8b and 8c, and a dynamic component identifiable from a step response or harmonic analysis.

In order to identify the dynamics and static gains $k_{x} D_{x}(s)$ and $k_{y} D_{y}(s)$, step reference inputs of $5 \mu \mathrm{m}$ were applied to the $x$ and $y$ axes of the system. An ARMAX paramet- ric identification technique was then used via MATLAB (Ljung (2008)) to obtain a model that matched the experimental data. The following second-order models were found to provide the best fit with the experimental data whilst avoiding an excessively high-order structure:

$$
\left\{\begin{array}{l}
k_{x} D_{x}(s)=\frac{5.6 \times 10^{-3}(s+5019)(s+5.9)}{(s+38.86)(s+4.3)} \\
k_{y} D_{y}(s)=\frac{6.7 \times 10^{-3}(s+5022)(s+15.5)}{(s+49.9)(s+10.5)}
\end{array}\right.
$$

A comparison between the simulated step response of the obtained model and the experimentally-obtained step response is shown in Fig. 9. As shown, there is a good correlation between the two results.

\section{IMC FEEDBACK CONTROL}

Having obtained a model of the linearized system, a feedback controller was designed in order to reject undesired factors such as mechanical cross-coupling and external disturbances, and to improve performance characteristics including tracking accuracy and response time. Such use of feedback control techniques for micro/nano-positioning systems has been demonstrated in a number of recent applications (Rakotondrabe et al. (2011); Lee et al. (2011); Bashash and Jalili (2009); Yong et al. (2010)).

\subsection{Control Principle}

The designed feedback controller is based on the principle of internal model control (IMC), with the controller containing an approximate model of the system. Fig. 10a depicts a block-diagram representation of the system including the fabricated MEMS nanopositioner, the feedforward controllers $\Gamma_{x}^{i}$ and $\Gamma_{y}^{i}$, and the IMC feedback scheme. In this representation, $x_{r}$ and $y_{r}$ are defined as the reference inputs for the closed-loop system.

The IMC feedback control system consists of the approximate plant models $\hat{G}_{x}(s)$ and $\hat{G}_{y}(s)$, and the controllers $C_{x}(s)$ and $C_{y}(s)$. The approximate models take the form:

$$
\left\{\begin{array}{l}
\hat{G}_{x}(s)=k_{x} D_{x}(s) \\
\hat{G}_{y}(s)=k_{y} D_{y}(s)
\end{array}\right.
$$

As described in the previous section, the linearized plant has the following structure:

$$
\left\{\begin{array}{l}
x=G_{x}(s) x_{r f}+d_{x} \\
y=G_{y}(s) y_{r f}+d_{y}
\end{array}\right.
$$

where $G_{x}(s)$ and $G_{y}(s)$ represent the actual plant, and $d_{x}$ and $d_{y}$ represent the mechanical cross-couplings, which are treated as disturbances. The model described in (6) differ from the real plants $G_{x}(s)$ and $G_{y}(s)$ only by the uncertainties in $k_{x} D_{x}(s)$ and $k_{y} D_{y}(s)$ respectively, which are compensated for by the IMC feedback controller. Fig. 10b shows the equivalent system block diagram when using the plant equation in (7). From these results, the following equation is derived:

$$
x=\frac{G_{x} C_{x}}{\left(1+C_{x}\left(G_{x}-\hat{G}_{x}\right)\right)} x_{r}+\frac{\left(1-\hat{G}_{x} C_{x}\right)}{\left(1+C_{x}\left(G_{x}-\hat{G}_{x}\right)\right)} d_{x}
$$

which describes the relationship between the reference input $x_{r}$, the output to be controlled $x$ and the disturbance 

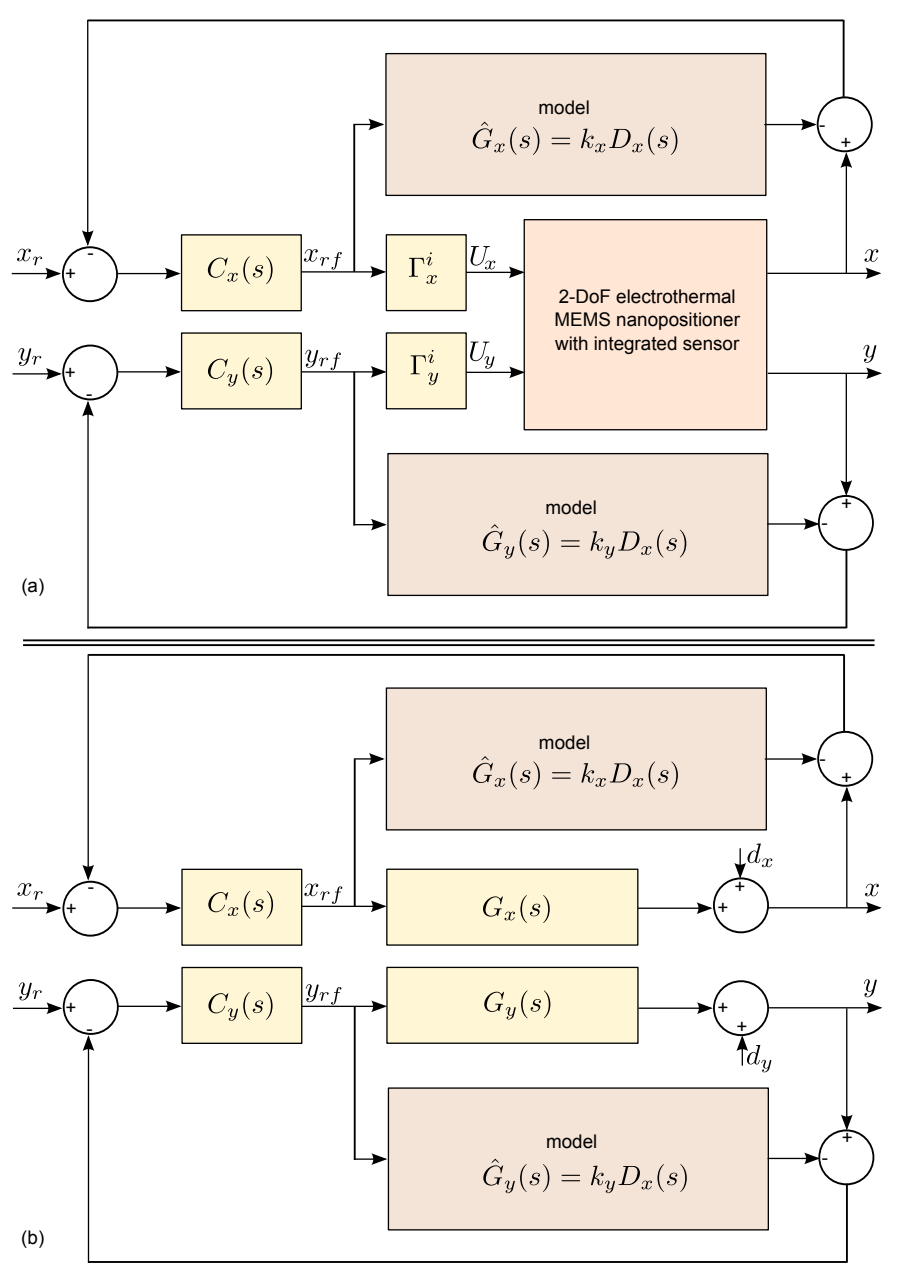

Fig. 10. Block diagram representation of the nanopositioning system including feedforward and feedback controllers.

$d_{x}$, which is assumed to consist of the mechanical crosscoupling and other external perturbations. In the following section the design of the $x$-axis controller $C_{x}(s)$ will be described based on this equation, with an identical process being assumed for the design of the $y$-axis controller.

\subsection{Controller Design}

Assume that the feedback controller is first chosen such that $C_{x}(s)=\frac{1}{\hat{G}_{x}(s)}$, which is then introduced into (8). Simplification of this expression yields:

$$
x=1 \cdot x_{r}+0 \cdot d_{x}
$$

which indicates that reference tracking is obtained $(x=$ $\left.x_{r}\right)$ and that disturbances are rejected $\left(x=0 \cdot d_{x}\right)$ for any reference input $x_{r}$, any disturbance $d_{x}$ and any approximate model $\hat{G}_{x}(s)$. The controller is then modified by adding a filter $F_{x}(s)$ such that:

$$
C_{x}(s)=\frac{1}{\hat{G}_{x}(s)} F_{x}(s)
$$

First consider the case where a perfect model of the plant is obtained, i.e. $G_{x}(s)=\hat{G}_{x}(s)$. Introducing this model into (10), and then into (8) yields:

$$
x=F_{x}(s) x_{r}+\left(1-F_{x}(s)\right) d_{x}
$$

According to (11), the filter $F_{x}(s)$ should be chosen as a reference model for the closed-loop transfer function $\frac{x(s)}{x_{r(s)}}$. For example, if the closed-loop response is desired that contains no overshoot and has a response time $\leq t_{x r}$, a first-order reference model with time constant $\tau_{x}=3 t_{x r}$ can be used, and the filter becomes:

$$
F_{x}(s)=\frac{1}{1+\tau_{x} s}
$$

Now consider that an imperfect plant model is obtained, i.e. $\hat{G}_{x}(s) \neq G_{x}(s)$. Substituting this into (8) yields:

$$
x=\frac{\frac{G_{x}}{\hat{G}_{x}} F_{x}}{\left(1+F_{x}\left(\frac{G_{x}}{\hat{G}_{x}}-1\right)\right)} x_{r}+\frac{\left(1-F_{x}\right)}{\left(1+F_{x}\left(\frac{G_{x}}{\hat{G}_{x}}-1\right)\right)} d_{x}
$$

By introducing the proposed filter (12) into (13) and letting $s=0$ (i.e. $t \rightarrow \infty$ ), the same result from (9) is obtained, implying that reference tracking and disturbance rejection is maintained in the new closed-loop system. However, the transient response of the system is dependent on the uncertainties between the approximate plant model $\hat{G}_{x}(s)$ and the real plant model $G_{x}(s)$.

For experimental testing, the filters $F_{x}(s)$ and $F_{y}(s)$ for the controllers $C_{x}(s)$ and $C_{y}(s)$ respectively were chosen such that the closed-loop system has a response time of $t_{x r}=t_{y r}=200 \mathrm{~ms}$. Using (10), (12), (6) and (5) the controllers are derived as:

$$
\left\{\begin{array}{c}
C_{x}(s)=\frac{(s+38.86)(s+4.3)}{5.6 \times 10^{-3}(s+5019)(s+5.9)\left(1+\frac{0.2}{3}\right)} \\
C_{y}(s)=\frac{(s+49.9)(s+10.5)}{6.7 \times 10^{-3}(s+5022)(s+15.5)\left(1+\frac{0.2}{3} s\right)}
\end{array}\right.
$$

\subsection{Experimental Testing of the Closed-Loop System}

The feedforward controllers $\Gamma_{x}^{i}$ and $\Gamma_{y}^{i}$ and the IMC feedback system were implemented to control the fabricated MEMS nanopositioner. A PC running MATLAB/Simulink was used to implement the controllers with a refresh time of $1 \mathrm{~ms}$, while a dSpace acquisition board was used to interface with the MEMS device (Fig. 11). An amplifier was used to drive the nanopositioner's actuators, while the measurements of $x$ - and $y$-axis stage displacement were carried out using the integrated electrothermal sensors.

The step response of the closed-loop system was analyzed, with Figs. 12a and $12 \mathrm{~b}$ showing the responses when a series of step references $x_{r}$ and $y_{r}$ are applied. It can be concluded from these plots that the outputs $x$ and $y$ are able to closely track these references, with the cross-coupling between axes successfully being rejected. Figs. 12c and $12 \mathrm{~d}$ show a zoomed view of the $x$ - and $y$-axis responses respectively to a step reference $x_{r}=$ $4 \mu \mathrm{m}$. It is observed that the the $x$-axis output has zero static error with a response time of around $100 \mathrm{~ms}$, which is a significant improvement on the open-loop response (approximately $200 \mathrm{~ms}$ ). In addition, the time taken to reject the cross-coupling from $x_{r}$ to $y$ is approximately $400 \mathrm{~ms}$, with the static error due to this disturbance approaching zero as $t \rightarrow \infty$, as predicted. Figs. $12 \mathrm{e}$ and $12 \mathrm{f}$ show zoomed views of of the responses $x$ and $y$ respectively to to a reference $y_{r}=4 \mu \mathrm{m}$. The $y$ output also shows 


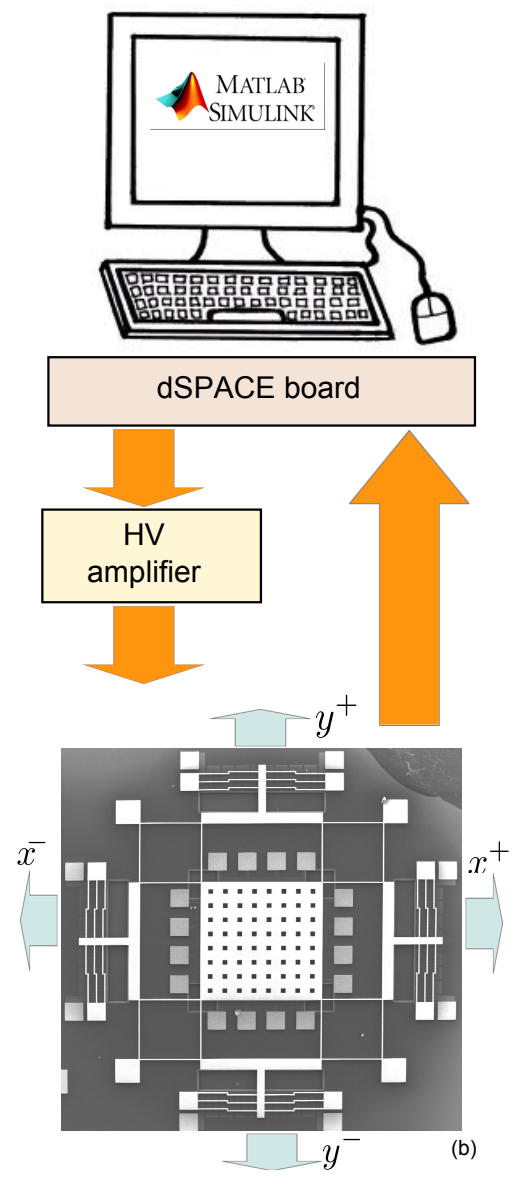

Fig. 11. The experimental setup.

zero static error, and the response time of around $120 \mathrm{~ms}$ is again an improvement on the open-loop performance. Rejection of the cross-coupling from $y_{r}$ to $x$ is observed, with the response time of this disturbance rejection being approximately $410 \mathrm{~ms}$.

A circular spatial reference was then applied to the system to test its ability to track a more complex reference in $2 \mathrm{D}$ space. This circular reference was generated by applying the following parametric functions to $x_{r}$ and $y_{r}$ :

$$
\left\{\begin{array}{l}
x_{r}(t)=R \cos \left(2 \pi f_{r} t\right) \\
y_{r}(t)=R \sin \left(2 \pi f_{r} t\right)
\end{array}\right.
$$

where $\mathrm{R}(\mu \mathrm{m})$ is the radius of the circle and $f_{r}(\mathrm{~Hz})$ is the frequency of rotation. Fig. 13 shows the experimental result, using a circular reference with radius $R=4 \mu \mathrm{m}$ and a frequency of $0.5 \mathrm{~Hz}$. As shown in the figure, the nanopositioner is able to closely track the reference, with further testing showing that similarly good tracking is achieved for frequencies lower than $5 \mathrm{~Hz}$.

\section{CONCLUSION}

This paper has described the characterization, modeling and control of a fabricated 2-DoF MEMS nanopositioner with integrated electrothermal actuators and sensors. The device's actuators, which are based on Z-shaped beams, are used to position a central stage via bidirectional motions along two in-plane axes. Experimental characterization shows that stage displacements in excess of $\pm 5 \mu \mathrm{m}$ are achievable along both the $x$ and $y$ axes, and that the
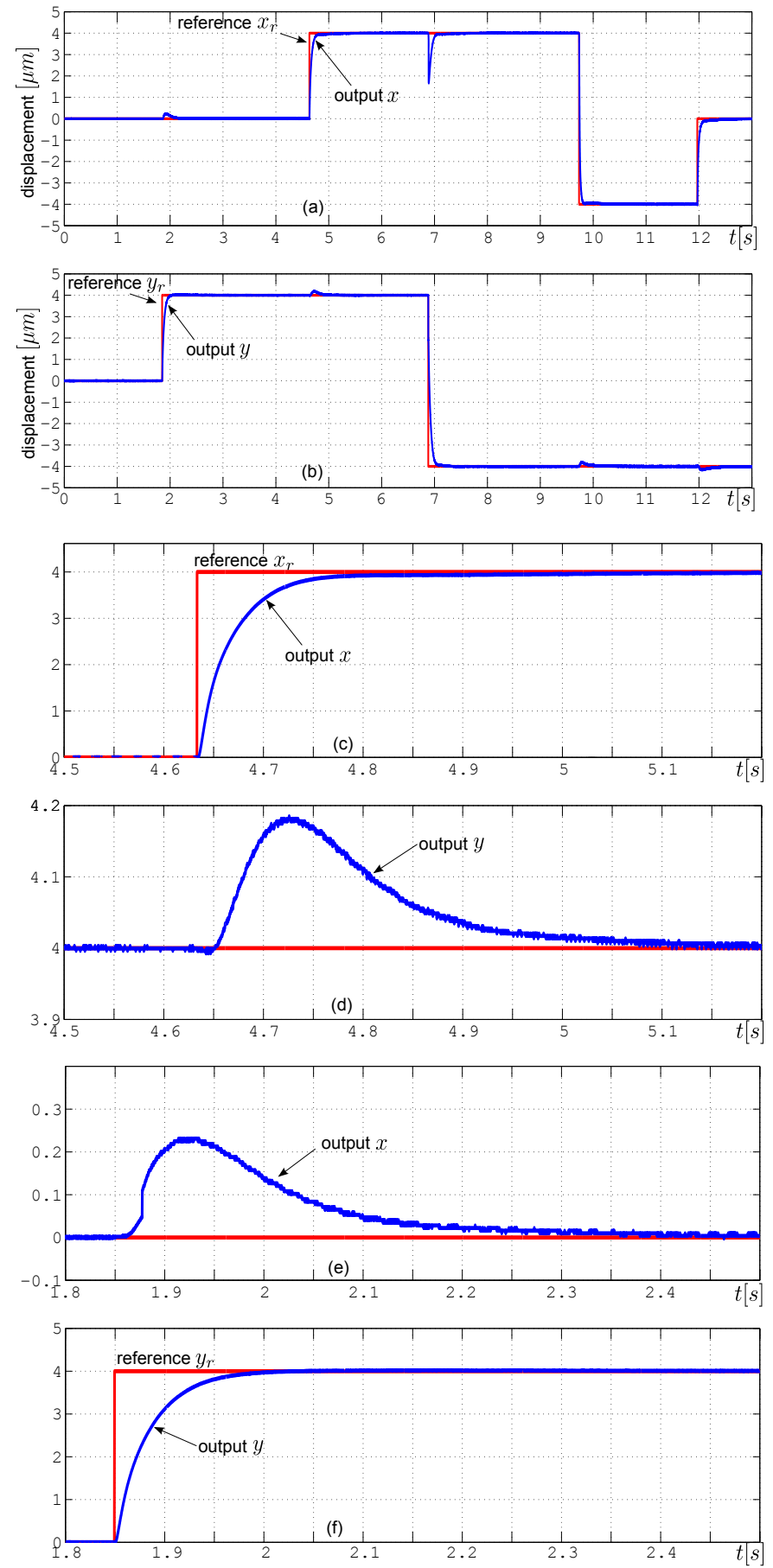

Fig. 12. Experimental step responses. (a) and (b): responses of the $x$ and $y$ axis respectively to a series of step references. (c) and (d): responses of $x$ and $y$ respectively to a step $x_{r}$. (e) and (f): responses of $x$ and $y$ respectively to a step $y_{r}$.

device has a response time of less than $300 \mathrm{~ms}$. A feedforward control scheme was to linearize the system, while the device's integrated displacement sensors were used to facilitate the implementation of a closed-loop controller. The feedback controller is based on the IMC principle, and is demonstrated to reject the mechanical cross-coupling between the axes of the device while improving the system's reference tracking ability and response time. These results 


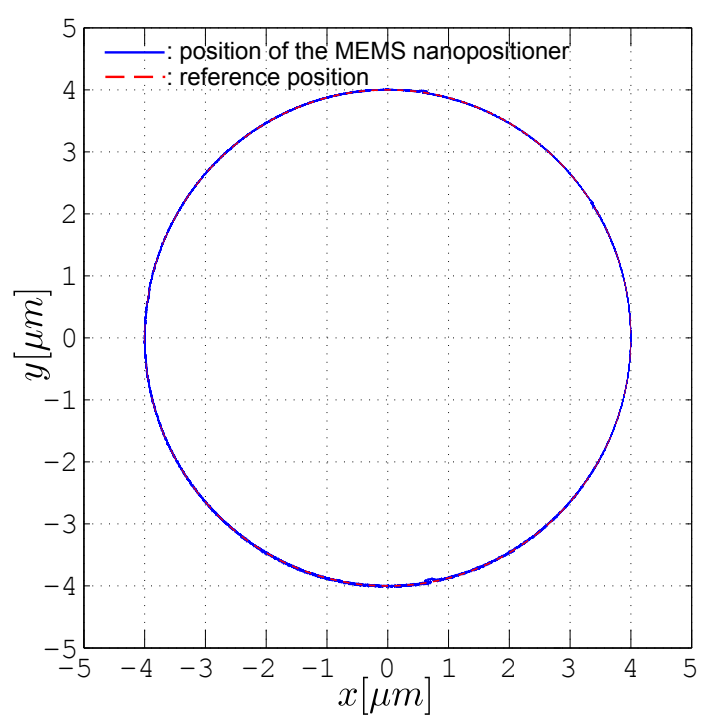

Fig. 13. System response to a circular reference input.

highlight the suitability of the fabricated MEMS system for nanopositioning applications.

\section{ACKNOWLEDGEMENTS}

The authors would like to thank Dr. Yong Zhu (Griffith University, Australia) for his contributions to the design of an earlier version of the MEMS device. The SEM images were obtained with the assistance of the University of Newcastle's Electron Microscope and X-Ray Unit. This work was supported by the Australian Research Council.

\section{REFERENCES}

Bashash, S. and Jalili, N. (2009). Robust adaptive control of coupled parallel piezo-flexural nanopositioning stages. IEEE/ASME Transactions on Mechatronics, 14(1), 1120.

Bazaei, A., Zhu, Y., Moheimani, S.O.R., and Yuce, M.R. (2012). Analysis of nonlinear phenomena in a thermal micro-actuator with a built-in thermal position sensor. IEEE Sensors Journal, 12(6), 1772-1784.

Bergna, S., Gorman, J., and Dagalakis, N. (2005). Design and modeling of thermally actuated MEMS nanopositioners. In Proceedings of the ASME IMECE, 561-568. Asme.

Binnig, G. and Rohrer, H. (1986). The scanning tunneling microscope. Scientific American, 253, 50-56.

Chen, S.C. and Culpepper, M.L. (2012). Design of contoured thermomechanical actuators and pulsing actuation to enhance dynamic performance. Journal of Microelectromechanical Systems, 21(2), 340-349.

Chow, J. and Lai, Y. (2009). Displacement sensing of a micro-electro-thermal actuator using a monolithically integrated thermal sensor. Sensors and Actuators A: Physical, 150(1), 137-143.

Cowen, A., Mahadevan, R., Johnson, S., and Hardy, B. (2009). MetalMUMPs Design Handbook.

Fowler, A.G., Laskovski, A.N., Hammond, A.C., and Moheimani, S.O.R. (2012). A 2-DOF electrostatically actuated MEMS nanopositioner for on-chip AFM. Journal of Microelectromechanical Systems, 21(4), 771-773.
Guan, C. and Zhu, Y. (2010). An electrothermal microactuator with Z-shaped beams. Journal of Micromechanics and Microengineering, 20(8), 085014.

Lantz, M., Rothuizen, H., Drechsler, U., Haberle, W., and Despont, M. (2007). A vibration resistant nanopositioner for mobile parallel-probe storage applications. Journal of Microelectromechanical Systems, 16(1), 130139.

Lantz, M.A., Binnig, G.K., Despont, M., and Drechsler, U. (2005). A micromechanical thermal displacement sensor with nanometre resolution. Nanotechnology, 16(8), 1089-1094.

Lee, C., Mohan, G., and Salapaka, S. (2011). 2dof control design for nanopositioning. In Control Technologies for Emerging Micro and Nanoscale Systems, 67-82. Springer Berlin / Heidelberg.

Ljung, L. (2008). System identification toolbox 7 user's guide. The Mathworks, Natick, MA USA.

Ouyang, J. and Zhu, Y. (2012). Z-shaped MEMS thermal actuators: Piezoresistive self-sensing and preliminary results for feedback control. Journal of Microelectromechanical Systems, 21(3), 596-604.

Pantazi, A., Lantz, M.A., Cherubini, G., Pozidis, H., and Eleftheriou, E. (2004). A servomechanism for a micro-electro-mechanical-system-based scanning-probe data storage device. Nanotechnology, 15(10), S612S621.

Pantazi, A., Sebastian, A., Cherubini, G., Lantz, M., Pozidis, H., Rothuizen, H., and Eleftheriou, E. (2007). Control of MEMS-based scanning-probe data-storage devices. IEEE Transactions on Control Systems Technology, 15(5), 824-841.

Rakotondrabe, M., Rabenorosoa, K., Agnus, J., and Chaillet, N. (2011). Robust feedforward-feedback control of a nonlinear and oscillating 2-dof piezocantilever. IEEE Transactions on Automation Science and Engineering, $8(3), 506-519$.

Sebastian, A. (2011). Systems and control approach to electro-thermal sensing. In E. Eleftheriou and S.O.R. Moheimani (eds.), Control Technologies for Emerging Micro and Nanoscale Systems, chapter 8, 137-152. Springer Berlin / Heidelberg.

Waterfall, T., Teichert, K., and Jensen, B. (2008). Simultaneous on-chip sensing and actuation using the thermomechanical in-plane microactuator. Microelectromechanical Systems, Journal of, 17(5), 1204-1209.

Yong, Y.K., Liu, K., and Moheimani, S.O.R. (2010). Reducing cross-coupling in a compliant xy nanopositioning stage for fast and accurate raster scanning. IEEE Transactions on Control Systems Technology, 18(5), 11721179

Zhu, Y., Moheimani, S.O.R., and Yuce, M.R. (2011a). Simultaneous capacitive and electrothermal position sensing in a micromachined nanopositioner. IEEE Electron Device Letters, 32(8), 1146-1148.

Zhu, Y., Bazaei, A., Moheimani, S.O.R., and Yuce, M.R. (2011b). Design, modeling, and control of a micromachined nanopositioner with integrated electrothermal actuation and sensing. Journal of Microelectromechanical Systems, 20(3), 711-719.

Zhu, Y., Moheimani, S.O.R., and Yuce, M.R. (2012). Bidirectional electrothermal actuator with Z-shaped beams. IEEE Sensors Journal, 12(7), 2508-2509. 